\title{
Large regular reflecting stars have no scalar field hair
}

\author{
Yan Peng ${ }^{\mathrm{a}}$ \\ School of Mathematical Sciences, Qufu Normal University, Qufu 273165, Shandong, China
}

Received: 12 December 2018 / Accepted: 29 March 2019 / Published online: 5 April 2019

(C) The Author(s) 2019

\begin{abstract}
We investigate the gravity system constructed with static scalar fields coupled to asymptotically flat regular reflecting stars. We consider the matter field's backreaction on the reflecting star. We analytically show that there is an upper bound on the radius of the reflecting star. When the star radius is above the bound, the reflecting star cannot support the existence of scalar field hairs. That means large reflecting stars cannot have scalar field hairs.
\end{abstract}

\section{Introduction}

According to the no hair theorem [1-4], the asymptotically flat black hole can be determined by the three conserved charges (the mass, angular momentum and charge of the black hole), see Refs. [5-19] and reviews [20,21]. The belief on the no hair theorem was partly based on the physical argument that exterior matter and radiation fields would either go away to the infinity or be swallowed by the black hole horizon. So no field can exist outside a black hole horizon, except when it is associated with the three conserved charges of black hole spacetimes.

Whether there is also no hair theorem in the horizonless spacetime is an interesting question to be answered. Recently, it was found that the no scalar hair behavior appears in the background of regular neutral reflecting stars. It was firstly proved in [22] that the static scalar field cannot exist outside asymptotically flat neutral compact reflecting stars without a horizon. When considering the nonminimal coupling between the massless scalar field and the gravity, it was shown that the no scalar hair theorem holds in certain range of the coupling model parameter [23]. And in the background of asymptotically dS gravity, it was found that the massive scalar, vector and tensor field cannot condense outside the regular neutral reflecting stars [24].

\footnotetext{
a e-mail: yanpengphy@163.com
}

Can the no hair theorem obtained in the neural horizonless gravity still holds in the charged horizonless spacetime? In the charged horizonless reflecting shell spacetime, it was analytically found that the static scalar field cannot exist outside the shell when the shell radius is large enough [25-27]. Moreover, it was shown that charged horizonless reflecting stars cannot support the existence of the scalar field when the star radius is above an upper bound [28-32]. And the no scalar hair behavior also appears in the large size regular star with other surface boundary conditions $[33,34]$. However, all the front discussion has been carried out in the probe limit. In this work, we try to generalize the discussion by considering the matter fields' backreaction on the metric and also examine whether there are upper bounds on hairy star radii.

This paper is structured as follows. In Sect. 2, we construct the gravity system composed of a static scalar field and a asymptotically flat regular reflecting star away from the probe limit. In Sect. 3, we analytically show that there is an upper bound for the reflecting star radius. Above the bound, the scalar field cannot condense outside the star surface. Our summary is in the last section.

\section{The gravity model composed of scalar fields and reflecting stars}

We choose to study static asymptotically flat regular reflecting star spacetimes. In Schwarzschild coordinates, the line element of the spherically symmetric star is $[35,36]$

$d s^{2}=-g(r) e^{-\chi(r)} d t^{2}+g^{-1} d r^{2}+r^{2}\left(d \varphi^{2}+\sin ^{2} \varphi d \phi^{2}\right)$.

The solutions $\chi(r)$ and $g(r)=1-\frac{2 m(r)}{r}$ only depend on the radial coordinate $\mathrm{r}$. Since we study the asymptotically flat spacetime, there is $\chi(r) \rightarrow 0$ and $m(r) \rightarrow M$ as $r \rightarrow \infty$, where $\mathrm{M}$ is the total mass of the spacetime. 
And the simple Lagrange density with scalar fields coupled to the charged star reads [37-39]

$\mathcal{L}=R-F^{M N} F_{M N}-\left|\nabla_{\alpha} \psi-q A_{\alpha} \psi\right|^{2}-\mu^{2} \psi^{2}$.

Here $\psi(r)$ is the scalar field and $A_{\alpha}$ corresponds to the Maxwell field. We define $\mathrm{R}, \mathrm{q}$ and $\mu$ as Ricci curvature, scalar field charge and scalar field mass respectively.

We assume that the Maxwell field has only the nonzero $t$ component in the form $A_{t}=\phi(r) d t$. Then the equation of the Maxwell field is [37-43]

$\phi^{\prime \prime}+\left(\frac{2}{r}+\frac{\chi^{\prime}}{2}\right) \phi^{\prime}-\frac{q^{2} \psi^{2}}{2 g} \phi=0$.

At the infinity, the asymptotical behavior of the electric potential is $\phi=-\frac{Q}{r}$, where $\mathrm{Q}$ is the total charge within the radius $r[28,29]$. The scalar field equation is [44-49]

$\psi^{\prime \prime}+\left(\frac{2}{r}-\frac{\chi^{\prime}}{2}+\frac{g^{\prime}}{g}\right) \psi^{\prime}+\left(\frac{q^{2} e^{\chi} \phi^{2}}{g^{2}}-\frac{\mu^{2}}{g}\right) \psi=0$.

And the metric equations are [37-39]

$\chi^{\prime}+r \psi^{\prime 2}+\frac{q^{2} r e^{\chi} \phi^{2} \psi^{2}}{g^{2}}=0$,

$g^{\prime}+\frac{g}{r}-\frac{1}{r}+r g\left[\frac{1}{2} \psi^{\prime 2}+\frac{e^{\chi} \phi^{\prime 2}}{g}+\frac{q^{2} e^{\chi} \phi^{2} \psi^{2}}{2 g^{2}}+\frac{\mu^{2} \psi^{2}}{2 g}\right]=0$.

In the linear case, we can simply set $\psi(r)=0$ in Eqs. (3), (5) and (6). The metric solutions are $g(r)=$ $1-\frac{2 M}{r}+\frac{Q^{2}}{r^{2}}, \chi=0$ and the Maxwell field outside the star is $\phi(r)=-\frac{Q}{r}$, where $Q$ is the star charge and $M$ is the star mass. We define $r_{s}$ as the star radius. In the limit case of $Q, M \ll r_{s}$ while $q Q$ fixed, non-trivial solutions of Eq. (4) were analytically obtained in [25-27]. The relations $Q, M \ll r_{s}$ and $g(r)=1-\frac{2 M}{r}+\frac{Q^{2}}{r^{2}}$ mean that exterior regions outside the star is assumed to be flat. We should point out that the presence of a charge $Q$ is crucial for the existence of the analytical solution in terms of Bessel functions. With the nonzero term $q Q, Q$ appears in the scalar field equation [25-27]. As a further step, for nonzero values $q, Q$ and $M$, we numerically obtained non-trivial solutions of Eq. (4) in the linear limit [28].

However, all front discussions have been carried out without scalar fields' backreaction. When considering scalar fields' backreaction on the metric, Eq. (4) is coupled with Eqs. (3), (5) and (6). In this case of nonlinear coupled equations, approaches in [25-28] fail and more precise numerical methods are needed. In this work, we analytically show that the non-trivial scalar field solution of nonlinear equations cannot exist when the star radius is above an upper bound. In other words, we prove no scalar field hair theorem for large reflecting stars in the nonlinear regime.
At the star surface, we take the scalar reflecting condition that the scalar field vanishes. Around the infinity, the scalar field asymptotically behaves in the form $\psi \sim A \cdot \frac{1}{r} e^{-\mu r}+B$. $\frac{1}{r} e^{\mu r}$, with $\mathrm{A}$ and $\mathrm{B}$ as integral constants. In order to obtain the physical solution, we set $B=0$. And the scalar field satisfies boundary conditions

$\psi\left(r_{s}\right)=0, \quad \psi(\infty)=0$.

The mass $m(r)$ within the radius $r$ is given by

$m(r)=\int_{0}^{r} 4 \pi r^{\prime 2} \rho\left(r^{\prime}\right) d r^{\prime}$,

where $\rho=-T_{t}^{t}$ is the energy density. For the probe charged star, there is $m(r)=M-\frac{Q^{2}}{2 r}$. In this work, we are interested in general asymptotically flat star with backreaction of matter fields. Considering the facts that the scalar field asymptotically goes to zero as $\psi \sim \frac{1}{r} e^{-\mu r}$ and charged Maxwell fields are associated with the $-\frac{Q}{r}$ asymptotic behavior, we deduce that the Maxwell field dominates the energy density and $\rho=-T_{t}^{t} \sim \frac{1}{r^{4}}$ around the infinity [35,50]. So there is

$m^{\prime}(r)=4 \pi r^{2} \rho(r) \rightarrow 0$

as $r \rightarrow \infty$.

\section{Upper bounds on radii of scalar hairy reflecting stars}

With a new function $\tilde{\psi}=\sqrt{r} \psi$, the Eq. (4) can be expressed as

$$
\begin{aligned}
& r^{2} \tilde{\psi}^{\prime \prime}+\left(r-\frac{r^{2} \chi^{\prime}}{2}+\frac{r^{2} g^{\prime}}{g}\right) \tilde{\psi}^{\prime} \\
& +\left(-\frac{1}{4}-\frac{r g^{\prime}}{2 g}+\frac{r^{2} q^{2} e^{\chi} \phi^{2}}{g^{2}}-\frac{\mu^{2} r^{2}}{g}\right) \tilde{\psi}=0 .
\end{aligned}
$$

According to (7), boundary conditions of $\tilde{\psi}$ are

$$
\tilde{\psi}\left(r_{s}\right)=0, \quad \tilde{\psi}(\infty)=0 .
$$

Then the function $\tilde{\psi}$ must possess at least one extremum point $r=r_{\text {peak }}$ above the star surface $r_{s}$. At this extremum point, there is the following characteristic relation

$\left\{\tilde{\psi}^{\prime}=0\right.$ and $\left.\tilde{\psi} \tilde{\psi}^{\prime \prime} \leqslant 0\right\} \quad$ for $\quad r=r_{\text {peak }}$.

With relations (10) and (12), we arrive at the following inequality

$$
-\frac{1}{4}-\frac{r g^{\prime}}{2 g}+\frac{r^{2} q^{2} e^{\chi} \phi^{2}}{g^{2}}-\frac{\mu^{2} r^{2}}{g} \geqslant 0 \text { for } r=r_{\text {peak }} .
$$

It can be transformed into

$\mu^{2} r^{2} g \leqslant r^{2} e^{\chi} q^{2} \phi^{2}-\frac{r g g^{\prime}}{2}-\frac{1}{4} g^{2} \quad$ for $\quad r=r_{\text {peak }}$. 
At the infinity, the asymptotical behaviors of the solutions are $\phi \rightarrow-\frac{Q}{r}$ and $e^{\chi} \rightarrow 1$. So a constant $R_{1}$ exists and for any $r \geqslant r_{s} \geqslant R_{1}$, the relations $-\frac{2 Q}{r}<\phi<-\frac{Q}{2 r}$ and $e^{\chi} \leqslant \frac{5}{4}$ hold. So there is

$$
\mu^{2} r^{2} g \leqslant 5 q^{2} Q^{2}-\frac{r g g^{\prime}}{2}-\frac{1}{4} g^{2} \text { for } r=r_{\text {peak }}
$$

According to (9), we have the relation

$$
\begin{aligned}
r g g^{\prime} & =r\left(1-\frac{2 m(r)}{r}\right)\left(1-\frac{2 m(r)}{r}\right)^{\prime} \\
& =r\left(1-\frac{2 m(r)}{r}\right)\left(\frac{2 m(r)}{r^{2}}-\frac{2 m^{\prime}(r)}{r}\right) \\
& =\left(1-\frac{2 m(r)}{r}\right)\left(\frac{2 m(r)}{r}-2 m^{\prime}(r)\right) \\
& \rightarrow\left(1-\frac{2 M}{r}\right)\left(\frac{2 M}{r}-2 m^{\prime}(r)\right) \rightarrow 0
\end{aligned}
$$

as $r \rightarrow \infty$. Then there is a constant $R_{2}$ and for any $r \geqslant$ $r_{s} \geqslant R_{2}$, the inequality $-2 q^{2} Q^{2}<r g g^{\prime}<2 q^{2} Q^{2}$ holds. So there is the relation

$\mu^{2} r^{2} g \leqslant 6 q^{2} Q^{2}-\frac{1}{4} g^{2} \leqslant 6 q^{2} Q^{2}$ for $r=r_{\text {peak }}$

with $r_{s} \geqslant R_{1}$ and $r_{s} \geqslant R_{2}$.

Since $g=1-\frac{2 m(r)}{r} \rightarrow 1-\frac{2 M}{r} \rightarrow 1$ as $r \rightarrow \infty$, there is a constant $R_{3}$ and for any $r \geqslant r_{s} \geqslant R_{3}$, there is the relation $g>\frac{1}{2}$. So we have

$\frac{\mu^{2} r_{s}^{2}}{2} \leqslant \frac{\mu^{2} r^{2}}{2} \leqslant \mu^{2} r^{2} g \leqslant 6 q^{2} Q^{2} \quad$ for $r=r_{\text {peak }}$

with $r_{s} \geqslant R_{1}, r_{s} \geqslant R_{2}$ and $r_{s} \geqslant R_{3}$.

We assume that star radii satisfy $r_{s} \geqslant R_{1}, r_{s} \geqslant R_{2}$ and $r_{s} \geqslant R_{3}$, otherwise we have an upper bound

$\mu r_{s} \leqslant \max \left\{\mu R_{1}, \mu R_{2}, \mu R_{3}\right\}$

According to (18) and $r_{\text {peak }} \geqslant r_{s}$, there is

$\mu r_{s} \leqslant \mu r_{\text {peak }} \leqslant 2 \sqrt{3} q Q$.

Our analysis shows that the hairy star radius is below the bounds (19) or (20). That is to say the hairy star radii can be divided into two cases

case 1: $\mu r_{s} \leqslant \max \left\{\mu R_{1}, \mu R_{2}, \mu R_{3}\right\}$

case 2: $\mu r_{s} \geqslant \max \left\{\mu R_{1}, \mu R_{2}, \mu R_{3}\right\}$ with $\mu r_{s} \leqslant 2 \sqrt{3} q Q$.

In all, we obtain upper bounds for hairy star radii as

$\mu r_{s} \leqslant \max \left\{2 \sqrt{3} q Q, \mu R_{1}, \mu R_{2}, \mu R_{3}\right\}$ with dimensionless quantities according to the symmetry of Eqs. (3)-(6)

$r \rightarrow k r, \quad \mu \rightarrow \mu / k, \quad q \rightarrow q / k$.

When the star radius is above the bound (21), the static scalar field cannot condense outside the static asymptotically flat spherically symmetric regular reflecting star. That means big reflecting star cannot support the existence of massive scalar field hair. In fact, there are also similar properties in black hole gravities. According to the no short hair conjecture, the hairy black hole horizon is below the upper bound $r_{H}<\frac{2}{3}(\eta)^{-1}$ with $\eta$ as the exterior field mass [51]. In fact, the numerical results also suggest that big black holes tend to have no massive hair [52].

\section{Conclusions}

We studied static massive scalar field condensations outside static asymptotically flat spherically symmetric regular reflecting stars. We constructed a complete gravity model by considering the matter field's backreaction on the background. We provided upper bounds on the star radius in the form $\mu r_{s} \leqslant \max \left\{2 \sqrt{3} q Q, \mu R_{1}, \mu R_{2}, \mu R_{3}\right\}$, where $\mu$ is the scalar field mass, $q$ is the charge coupling parameter, $Q$ is the total charge and $R_{i}$ depends on the gravity theories. When the star radius is above the bound, the static massive scalar field cannot condense outside the reflecting star. That means the large regular reflecting star cannot have massive scalar field hairs in the asymptotically flat gravity.

Acknowledgements We would like to thank the anonymous referee for the constructive suggestions to improve the manuscript. This work was supported by the Shandong Provincial Natural Science Foundation of China under Grant No. ZR2018QA008.

Data Availability Statement This manuscript has no associated data or the data will not be deposited. [Authors' comment: This work is based on analytical study. So there is no data.]

Open Access This article is distributed under the terms of the Creative Commons Attribution 4.0 International License (http://creativecomm ons.org/licenses/by/4.0/), which permits unrestricted use, distribution, and reproduction in any medium, provided you give appropriate credit to the original author(s) and the source, provide a link to the Creative Commons license, and indicate if changes were made. Funded by SCOAP ${ }^{3}$.

\section{References}

1. J.D. Bekenstein, Transcendence of the law of baryon-number conservation in black hole physics. Phys. Rev. Lett. 28, 452 (1972)

2. J.E. Chase, Event horizons in static scalar-vacuum space-times. Commun. Math. Phys. 19, 276 (1970)

3. C. Teitelboim, Nonmeasurability of the baryon number of a blackhole. Lett. Nuovo Cimento 3, 326 (1972) 
4. R. Ruffini, J.A. Wheeler, Introducing the black hole. Phys. Today 24, 30 (1971)

5. S. Hod, Stationary scalar clouds around rotating black holes. Phys. Rev. D 86, 104026 (2012)

6. S. Hod, Stationary resonances of rapidly-rotating Kerr black holes. Euro. Phys. J. C 73, 2378 (2013)

7. S. Hod, Kerr-Newman black holes with stationary charged scalar clouds. Phys. Rev. D 90, 024051 (2014)

8. S. Hod, The large-mass limit of cloudy black holes. Class. Quant. Grav. 32, 134002 (2015)

9. S. Hod, The superradiant instability regime of the spinning Kerr black hole. Phys. Lett. B 758, 181 (2016)

10. C.A.R. Herdeiro, E. Radu, Kerr black holes with scalar hair. Phys. Rev. Lett. 112, 221101 (2014)

11. C.L. Benone, L.C.B. Crispino, C. Herdeiro, E. Radu, KerrNewman scalar clouds. Phys. Rev. D 90, 104024 (2014)

12. C. Herdeiro, E. Radu, H. Runarsson, Non-linear QQ-clouds around Kerr black holes. Phys. Lett. B 739, 302 (2014)

13. C. Herdeiro, E. Radu, Construction and physical properties of Kerr black holes with scalar hair. Class. Quant. Grav. 32, 144001 (2015)

14. Yan Peng, Hair mass bound in the black hole with non-zero cosmological constants. Phys. Rev. D 98, 104041 (2018)

15. Yan Peng, Hair distributions in noncommutative Einstein-BornInfeld black holes. Nucl. Phys. B 941, 1-10 (2019)

16. Yan Peng, The extreme orbital period in scalar hairy kerr black holes. Phys. Lett. B 792, 1-3 (2019)

17. J.C. Degollado, C.A.R. Herdeiro, Stationary scalar configurations around extremal charged black holes. Gen. Relat. Gravit. 45, 2483 (2013)

18. P.V.P. Cunha, C.A.R. Herdeiro, E. Radu, H.F. Runarsson, Shadows of Kerr black holes with scalar hair. Phys. Rev. Lett. 115, 211102 (2015)

19. Y. Brihaye, C. Herdeiro, E. Radu, Inside black holes with synchronized hair. Phys. Lett. B 760, 279 (2016)

20. J.D. Bekenstein, Black hole hair: 25-years after, arXiv:gr-qc/9605059

21. Carlos A.R. Herdeiro, Eugen Radu, Asymptotically flat black holes with scalar hair: a review. Int. J. Mod. Phys. D 24(09), 1542014 (2015)

22. S. Hod, No-scalar-hair theorem for spherically symmetric reflecting stars. Phys. Rev. D 94, 104073 (2016)

23. S. Hod, No nonminimally coupled massless scalar hair for spherically symmetric neutral reflecting stars. Phys. Rev. D 96, 024019 (2017)

24. Srijit Bhattacharjee, Sudipta Sarkar, No-hair theorems for a static and stationary reflecting star. Phys. Rev. D 95, 084027 (2017)

25. S. Hod, Charged massive scalar field configurations supported by a spherically symmetric charged reflecting shell. Phys. Lett. B 763, 275 (2016)

26. S. Hod, Marginally bound resonances of charged massive scalar fields in the background of a charged reflecting shell. Phys. Lett. B 768, 97-102 (2017)

27. Yan Peng, Bin Wang, Yunqi Liu, Scalar field condensation behaviors around reflecting shells in Anti-de Sitter spacetimes. Eur. Phys. J. C 78(8), 680 (2018)

28. Yan Peng, Scalar field configurations supported by charged compact reflecting stars in a curved spacetime. Phys. Lett. B 780, 144148 (2018)

29. Shahar Hod, Charged reflecting stars supporting charged massive scalar field configurations. Eur. Phys. J. C 78, 173 (2017)
30. Yan Peng, Static scalar field condensation in regular asymptotically AdS reflecting star backgrounds. Phys. Lett. B 782, 717-722 (2018)

31. Yan Peng, On instabilities of scalar hairy regular compact reflecting stars. JHEP 10, 185 (2018)

32. Yan Peng, Hair formation in the background of noncommutative reflecting stars. Nucl. Phys. B 938, 143-153 (2019)

33. Yan Peng, Scalar condensation behaviors around regular Neumann reflecting stars. Nucl. Phys. B 934, 459-465 (2018)

34. Y. Peng, No hair theorem for spherically symmetric regular compact stars with Dirichlet boundary conditions. arXiv:1901.11415 [gr-qc]

35. D. Núñez, H. Quevedo, D. Sudarsky, Black holes have no short hair. Phys. Rev. Lett. 76, 571 (1996)

36. S. Hod, Hairy black holes and null circular geodesics. Phys. Rev. D 84, 124030 (2011). arXiv:1112.3286 [gr-qc]

37. Pallab Basu, Chethan Krishnan, P.N. Bala Subramanian, Hairy black holes in a box. JHEP 11, 041 (2016)

38. Yan Peng, Studies of a general flat space/boson star transition model in a box through a language similar to holographic superconductors. JHEP 1707, 042 (2017)

39. Yan Peng, Bin Wang, Yunqi Liu, On the thermodynamics of the black hole and hairy black hole transitions in the asymptotically flat spacetime with a box. Eur. Phys. J. C 78(3), 176 (2018)

40. S.A. Hartnoll, C.P. Herzog, G.T. Horowitz, Holographic superconductors. JHEP 0812, 015 (2008)

41. Hua Bi Zeng, Yu. Tian, Zhe Yong Fan, Chiang-Mei Chen, Nonlinear transport in a two dimensional holographic superconductor. Phys. Rev. D 93, 121901 (2016)

42. X.H. Ge, B. Wang, S.F. Wu, G.H. Yang, Analytical study on holographic superconductors in external magnetic field. JHEP 08, 108 (2010)

43. Yi Ling, Peng Liu, Wu Jian-Pin, Note on the butterfly effect in holographic superconductor models. Phys. Lett. B 768, 288 (2017)

44. S.A. Hartnoll, C.P. Herzog, G.T. Horowitz, Holographic superconductors. JHEP 12, 015 (2008)

45. Nicolas Sanchis-Gual, Juan Carlos Degollado, Pedro J. Montero, Jos A. Font, Carlos Herdeiro, Explosion and final state of an unstable Reissner-Nordström black hole. Phys. Rev. Lett 116, 141101 (2016)

46. Sam R. Dolan, Supakchai Ponglertsakul, Elizabeth Winstanley, Stability of black holes in Einstein-charged scalar field theory in a cavity. Phys. Rev. D 92, 124047 (2015)

47. Pallab Basu, Chethan Krishnan, P.N. Bala Subramanian, Phases of global AdS black holes. JHEP 06, 139 (2016)

48. Marek Rogatko, Karol I. Wysokinski, Viscosity of holographic fluid in the presence of dark matter sector. JHEP 1608, 124 (2016)

49. Wu Chen, Xu Renli, Decay of massive scalar field in a black hole background immersed in magnetic field. Eur. Phys. J. C 75(8), 391 (2015)

50. Yan Peng, Qiyuan Pan, Yunqi Liu, A general holographic insulator/superconductor model with dark matter sector away from the probe limit. Nucl. Phys. B 915, 69-83 (2017)

51. Darío Núñez, Hernando Quevedo, Daniel Sudarsky, Black holes have no short hair. Phys. Rev. Lett. 76, 571-574 (1996)

52. Takashi Torii, Kei-Ichi Maeda, Takashi Tachizawa, Non-Abelian black holes and catastrophe theory. 1. Neutral type. Phys. Rev. D 51, 1510-1524 (1995) 